\title{
Microcatheter Originating Debris during Neuroendovascular Procedures: Mechanism of Dislodgement and Its Prevention
}

\author{
(D). Kan, (D) K. Karagiozov, (DS. Ito, (D)S. Sato, and (D) Y. Murayama
}

\begin{abstract}
SUMMARY: Embolic material dislodgement from microcatheters can potentially induce subclinical brain damage as evidenced by a delayed enhanced or other type of lesions. Some of the most frequently used microcatheters were investigated in vitro in different setups and combinations with different port insertions and rotating hemostatic valves. It was found that side port application increases injury to the catheter surface and debris dislodgement by conflicting with internal ledges in rotating hemostatic valves. This initial observation suggests the need for measures to remove the produced debris during such procedures.
\end{abstract}

ABBREVIATIONS: DEL = delayed enhanced lesion; FTIR = Fourier-transform infrared; RHV = rotating hemostatic valve

ntraprocedural embolization by coating material particles from medical devices, especially stent devices, ${ }^{1,2}$ and nickel allergy reaction to these particles, ${ }^{3,4}$ have been presumed to be the potential cause of delayed enhanced lesions (DELs) after endovascular surgery, but these have also been reported after the double-catheter technique ${ }^{5}$ and balloon-assisted coil embolization ${ }^{6}$ with multiple-microcatheter usage. Therefore, an in vitro setup of dualmicrocatheter access via 2 single-port, rotating hemostatic valves (RHVs) connected one to the other via the side port was investigated for debris dislodgement.

\section{MATERIALS AND METHODS \\ Microcatheters and Devices}

The Excelsior SL-10 microcathter (Stryker) was evaluated by in vitro simulation of its application in different device configurations during endovascular procedures, by comparing it with 3 similar types of microcatheters and between 2 similar RHV devices.

For hydrophilic coating-integrity study before and after interactions with the RHV, the Excelsior SL-10 was stained with a $0.1 \%$ aqueous solution of Congo red for 60 minutes and dried for

Received April 15, 2020; accepted after revision June 15.

From the Departments of Neurosurgery (I.K., K.K., Y.M.) and Pathology (S.I., S.S.), The Jikei University Hospital, Tokyo, Japan.

This work was supported by an educational research grant to our department from Stryker.

Please address correspondence to Issei Kan, MD, Department of Neurosurgery, The Jikei University Hospital, 3-25-8 Nishi-Shinbashi, Minato-ku, 105-8461 Tokyo, Japan; e-mail: isseikan@gmail.com

Indicates article with supplemental on-line photos.

http://dx.doi.org/10.3174/ajnr.A6723
24 hours, $^{7}$ and the Headway 17 (Terumo) and Echelon 10 (Medtronic) microcatheters were stained with a $0.1 \%$ aqueous solution of Toluidine blue.

Two single-port RHVs (Abbott 0.096-inch model 23242 RHV; Abbott Vascular) (AbbottRHV) were connected in series, via the side port (On-line Fig 1). Sequentially, we tested an SL-10 insertion (with a CHIKAI 14 guidewire; Asahi Intecc) separately through the attached RHV straight port or the side ports; 2 microcatheters under the same conditions, a Headway 21 (Terumo) or Prowler 21 (Codman \& Shurtleff) microcatheter through the straight port and the SL-10 through the side port; and finally an SL-10 without a guidewire (Table). Next a combination of Headway 17 and Echelon 10 were tested. Finally, a Gateway Advantage Y Adaptor model H74904482012 (Boston Scientific) (BostonRHV) was compared with the Abbott model.

The inside of the RHV was flushed with saline as during routine surgical procedures on a single insertion and extraction through the available port, and the skived material from microcatheters was collected from the flush fluid in a $0.45-\mu \mathrm{m}$ filter. Three neurointerventionalists including the first author independently assessed the amount of debris, rating it semiquantitatively in 3 grades: -, none; + , any identifiable; and ++ , substantial amount/bulk of debris (On-line Fig 2B).

After extraction from the RHV, the length of the stained microcatheter from the tip to $1000 \mathrm{~mm}$ proximally was photographed using a stereomicroscope. For microscopic testing, the debris from an unstained microcatheter was collected in saline and sent to the pathology department, where Papanicolaou and hematoxylin-eosin stains were applied.

The samples collected in the $0.45-\mu \mathrm{m}$ filters were directly analyzed using Fourier-transform infrared (FTIR) spectroscopy with 


\begin{tabular}{|c|c|c|c|c|c|}
\hline \multirow[b]{2}{*}{ Step } & \multirow[b]{2}{*}{ Side Port } & \multirow[b]{2}{*}{ Straight Port } & \multirow[b]{2}{*}{ RHV } & \multicolumn{2}{|c|}{ Amount of Debris } \\
\hline & & & & Insertion & Extraction \\
\hline \multirow[t]{5}{*}{$1 \mathrm{SL}-10$ Evaluation } & None & SL-10 & Abbott & - & - \\
\hline & SL-10 & None & Abbott & + & ++ \\
\hline & SL-10 ${ }^{a, b}$ & Headway 21 & Abbott & + & ++ \\
\hline & SL-10 & Prowler 21 & Abbott & + & ++ \\
\hline & SL-10 without guidewire & Prowler 21 & Abbott & - & - \\
\hline \multirow{2}{*}{$\begin{array}{l}2 \text { Microcatheter comparison } \\
\text { (similar MC to SL-10) }\end{array}$} & Headway $17^{a}$ & Headway 21 & Abbott & + & ++ \\
\hline & Echelon $10^{\mathrm{a}}$ & Headway 21 & Abbott & + & ++ \\
\hline 3 RHV comparison (other RHV) & $S L-10^{b}$ & Headway 21 & Boston Scientific & - & + \\
\hline
\end{tabular}

Note:-MC indicates microcatheter; - for amount of debris, none; + , identifiable; ++ , substantial, "None" under Side Port and Straight Port, absence of device use.

${ }^{a}$ Microcatheter comparison.

${ }^{b} \mathrm{RHV}$ comparison.

a Thermo-Nicolet 6700 FTIR spectrometer (attenuated total reflection mode; Thermo Electron) and a germanium crystal with a typical penetration depth of $\sim 1 \mu \mathrm{m}$. The analytic spot size was approximately $100 \times 100 \mu \mathrm{m}$. OMNIC Series Software (ThermoFisher) was used for the data analysis.

The pulling resistance force for SL-10 was evaluated with and without a guidewire through the 2 different types of RHVs using Digital Force Gauge DS2-20N (Imada, Aichi, Japan).

Both RHVs were imaged using a MicroCT Scanner NSI X25 (GE Healthcare) for cross-sectional views (14.7- $\mu \mathrm{m}$ voxel). High-resolution conebeam DynaCT RHV images (Artis Q biplane; Siemens) with the microcatheter inserted were acquired to measure the curvature radius of the microcatheter and the angle between the RHV side and straight ports.

\section{RESULTS}

All single-test combinations with side port SL-10 use (alone or through Headway 21 or Prowler 21) yielded some amount of debris at insertion and much more at extraction. The straight port SL-10 insertion and the SL-10 without a guidewire or together with the Prowler 21 did not produce debris.

The double-microcatheter application (Headway17/Headway21, Echelon10/Headway21) produced smaller amounts of debris at insertion and more significant amounts at extraction.

On a single experiment performed, the AbbottRHV produced more debris than the BostonRHV both on insertion and extraction.

All microcatheters inserted through the additional side port RHV (with and without a microcatheter in the main port) demonstrated damaged or skived coating $110 \mathrm{~mm}$ from the microcatheter tip (On-line Figs $1 A$ and $2 A$ ), and microscopically stained debris resembled the microcatheter coating.

FTIR debris analysis from the Excelsior SL-10 detected polyvinylpyrrolidone and a sulfonate salt similar to sodium lignosulfonate, and from the Headway 17, an organic acid salt similar to sodium alginate with trace of silicone species. Similar substances were detected for the Echelon 10 microcatheter. These results were all indicative of chemical structural components of catheter coating materials, mentioned in the corresponding documents accompanying the devices.

AbbottRHV produced 16-gram-force resistance with the guidewire and 3-gram-force without it, while for BostonRHV these values were 4 gram-force and 1 gram-force respectively, 3 to 4 times difference between RHVs and with/without the guidewire.

The CT scans of both AbbottRHV and BostonRHV showed a small ledge (protrusion) at the junction of the side and straight ports (On-line Fig 1C). The microcatheter inserted through the side port has to follow a tighter turn that requires direct firm contact with the ledge.

\section{DISCUSSION}

This in vitro study confirms that coating skiving of several types of stained-in-advance microcatheters will release debris when inserted through the side ports of RHVs. Some authors already have suggested that concurrent use of multiple microcatheters may cause DEL, blaming intracatheter friction and skiving of the microcatheter coating. ${ }^{1,5,8}$

We selected the widely applied method of multiple-microcatheter use and explored its course through the RHV to find more severe coating damage with the AbbottRHV. It can be assumed that differences in geometries between RHVs with the presence of a ledge inside creating friction resistance are probably the reason for this observation.

Biopsy-proved existence of foreign body emboli after endovascular treatment of intracranial aneurysms has already been reported, and pathologic findings have shown foreign body granuloma reaction with inflammatory cell infiltration. ${ }^{1,6,9}$ In the center of the granuloma, blue-gray nonpolarized material was frequently observed with $\mathrm{H} \& \mathrm{E}$ staining, and it appeared to be polyvinylpyrrolidone catheter coating. ${ }^{1,10}$

Most articles reporting DELs (cerebral leptomeningeal enhancement with perilesional edema seen after endovascular treatment of cerebral aneurysms) refer to the use of stent-assisted coil embolization or flow diverters. ${ }^{1-4}$ Speculated potential causes were polyglycolic lactic acid polymer, ${ }^{11}$ hydrophilic coating, ${ }^{1,12-14}$ and nickel allergy. ${ }^{3}$ However, DEL occurrence following coil embolization as well as balloon-assisted coil embolization have also been reported. $2,5,6,15$

A continuous skiving of all microcatheter coating after staining was observed from 110 to $1000 \mathrm{~mm}$ from the tip, and the obtained debris was similar to that in previous reports. ${ }^{1,10}$ The microcatheter distal tip is more flexible than its proximal sections, and that feature may be the cause for not observing skived 
coating most distally as impact is reduced. Insertion with the guidewire reduced microcatheter flexibility and increased the debris amount.

On the basis of this study, removing the guidewire before microcatheter extraction and aspiration of the guiding catheter content at the time of microcatheter extraction to reduce coating debris discharge into the circulation are suggested because the constant infusion through the port will inject debris into the circulation. Internal lumen modification of RHVs to reduce the mechanical conflict of side port channels could be helpful.

As a preliminary investigation, this study inherently contains limitations. First, currently, it is not confirmed that skived coating causes DEL. Next, there is no evidence for or against changes induced by the dyes used on the catheter coating, and finally, there are many different brands and models of RHVs and microcatheters currently available in different clinical settings; therefore, further investigation of these devices is warranted.

\section{CONCLUSIONS}

This preliminary study shows that a microcatheter insertion through an RHV side port may induce coating damage and debris dislodgement. Further investigation is warranted into dualmicrocatheter access using different device combinations, and redesigning to minimize microcatheter coating damage and debris release. Measures to counteract this issue are necessary.

\section{ACKNOWLEDGMENTS}

We are very grateful for the contribution of Dr. Toshihiro Ishibashi and Dr. Tomonobu Kodama for the independent blind assessment of the amount of debris produced. Stryker provided the methodology and performed the FTIR and MicroCT investigations, with the catheter staining/debris collection technique in this study.

Disclosures: Yuichi Murayama-UNRELATED: Consultancy: Stryker, Kaneka Medix, Tokai Medical Products; Grants/Grants Pending: Stryker, Comments: An educational research grant to our department from Stryker was used, in part, to support this study.* *Money paid to the institution.

\section{REFERENCES}

1. Shapiro M, Ollenschleger MD, Baccin C, et al. Foreign body emboli following cerebrovascular interventions: clinical, radiographic, and histopathologic features. AJNR Am J Neuroradiol 2015;36:2121-26 CrossRef Medline

2. Cruz JP, Marotta T, O'Kelly C, et al. Enhancing brain lesions after endovascular treatment of aneurysms. AJNR Am J Neuroradiol 2014;35:1954-58 CrossRef Medline

3. Park HS, Nakagawa I, Yokoyama S, et al. Nickel-associated delayed multiple white matter lesions after stent-assisted coil embolization of intracranial unruptured aneurysm. J Neurointerv Surg 2018;10:e1 CrossRef Medline

4. Tsang AC, Nicholson P, Pereira VM. Nickel-related adverse reactions in the treatment of cerebral aneurysms: a literature review. World Neurosurg 2018;115:147-53 CrossRef Medline

5. Oh SW, Shin NY, Lee HJ, et al. Delayed enhancing lesions after coil embolization of aneurysms: clinical experience and benchtop analyses. J Neurointerv Surg 2017;9:1243-47 CrossRef Medline

6. Shotar E, Law-Ye B, Baronnet-Chauvet F, et al. Non-ischemic cerebral enhancing lesions secondary to endovascular aneurysm therapy: nickel allergy or foreign body reaction? Case series and review of the literature. Neuroradiology 2016;58:877-85 CrossRef Medline

7. Babcock DE, Hergenrother RW, Craig DA, et al. In vivo distribution of particulate matter from coated angioplasty balloon catheters. Biomaterials 2013;34:3196-205 CrossRef Medline

8. Ikemura A, Ishibashi T, Otani K, et al. Delayed leukoencephalopathy: a rare complication after coiling of cerebral aneurysms. AJNR Am J Neuroradiol 2020;41:286-92 CrossRef Medline

9. Hu YC, Deshmukh VR, Albuquerque FC, et al. Histopathological assessment of fatal ipsilateral intraparenchymal hemorrhages after the treatment of supraclinoid aneurysms with the Pipeline Embolization Device. J Neurosurg 2014;120:365-74 CrossRef Medline

10. Fealey ME, Edwards WD, Giannini C, et al. Complications of endovascular polymers associated with vascular introducer sheaths and metallic coils in 3 patients, with literature review. Am J Surg Pathol 2008;32:1310-16 CrossRef Medline

11. Mehta RI, Mehta RI, Fishbein MC, et al. Intravascular polymer material after coil embolization of a giant cerebral aneurysm. Hum Pathol 2009;40:1803-07 CrossRef Medline

12. Mehta RI, Mehta RI. Hydrophilic polymer embolism: an update for physicians. Am J Med 2017;130:e287-90 CrossRef Medline

13. Stanley JRL, Tzafriri AR, Regan K, et al. Particulates from hydrophilic-coated guiding sheaths embolise to the brain. Eurointervention 2016;11:1435-41 CrossRef Medline

14. Mehta RI, Mehta RI, Solis OE, et al. Hydrophilic polymer emboli: an under-recognized iatrogenic cause of ischemia and infarct. Mod Pathol 2010;23:921-30 CrossRef Medline

15. Skolarus LE, Gemmete JJ, Braley T, et al. Abnormal white matter changes after cerebral aneurysm treatment with polyglycolic-polylactic acid coils. World Neurosurg 2010;74:640-44 CrossRef Medline 